\title{
Simulation of Magnetizing Inrush Current of Three-Phase Three-Legged Transformers by Direct Measurement of Coils Inductance
}

\author{
C. Sánchez-Martos ${ }^{1}$, M. Gómez-González², F. Jurado² \\ ${ }^{1}$ Department of Electrical Engineering \\ University of Jaén \\ Campus of Lagunillas - 23071 Jaén (Spain) \\ e-mail: csanchez@ujaen.es \\ ${ }^{2}$ Department of Electrical Engineering \\ University of Jaén \\ EPS Linares - 23700 Jaén (Spain) \\ e-mail: mggonzal@ujaen.es, fjurado@ujaen.es
}

\begin{abstract}
This paper presents a method for simulating magnetizing inrush current for three-phase three-legged transformers. A method is showed for easy and fast needed transformer parameters obtaining. It is shown that magnetizing inrush current is strongly dependent on transformer core saturation, and a straightforward method is presented for taking into account core saturation. Finally, a real transformer simulation results are presented and compared with those obtained by direct measure of magnetizing inrush current, discussing method suitability.
\end{abstract}

\section{Key words}

Simulation, three-legged transformer, magnetizing inrush currents.

\section{Introduction}

Since incorporation of digital protection for three-phase transformers, it was shown clearly the needed for having accurate algorithms for transformers magnetizing inrush current.

Since forties of XX century works for explaining phenomena associated with magnetizing inrush current have been conducted, trying to establish a mathematical formulation allowing calculation of a theoretical value and their evolution. For the case of three-phase transformers, by far the most widely used, the associated phenomena for magnetizing inrush current are much more complex than in the case of single-phase transformers.

At present there are several tools that allow the simulation of electromagnetic transients. In [1] a study of several simulation tools magnetizing current through programs EMPT/ATP, PSCAD and Matlab/Simulink is presented. The authors conclude by confirming the correct answer of the above tools for application to the study of transformer differential protection.

However, the development of a transformer accurate model is very complex due to the large number of different designs of cores and that several transformer parameters are nonlinear. In [2] a review of the proposed models of transformers for transient behavior at low frequency is presented. The authors conclude that it is difficult to develop such methods due to the large variety of designs for three-phase transformer cores, the frequency dependent behavior and the nonlinearity of many parameters of the transformer, and the acquisition and determination of some of the parameters. The development of a transformer model is a complex task.

\section{Magnetizing inrush current for three- phase transformers}

There have been several studies for the digital simulation of the magnetizing inrush current in threephase transformers. In [3] the authors present a study of the determination of the magnetizing inrush current by two methods, the first based on the sequence impedances and the second based on the mutual coupling.

In the first case it is assumed that the system is equivalent to three one-phase transformers magnetically decoupled, so the accuracy of the results is certainly limited, and the determination of the parameters is laborious.

The mutual coupling method does take into account all the magnetic coupling between phases, and is simpler, although the accuracy depends on the values of mutual inductances which are not linear. 
In [4] the authors present a simulation of the magnetizing inrush current that takes into account the magnetic characteristics of the core, but the core parameters are internal dimensions, which greatly complicates its application to real transformers already mounted. This paper presents a method based on the method of the magnetic coupling designed for the determination of the magnetizing inrush current for threephase three-legged transformer using a simple method of obtaining the precise parameters of the transformer.

\section{Transformer model}

Figure 1 shows the core of a three-phase three-legged transformer.

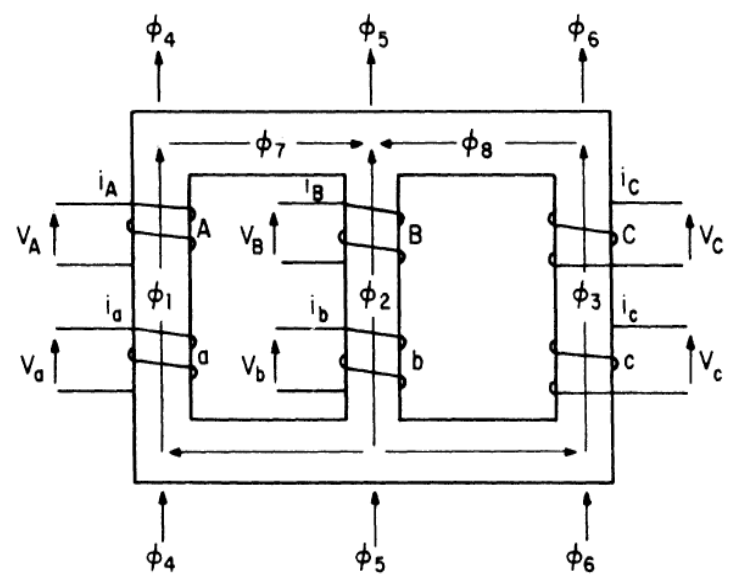

Fig. 1 Core of a three-phase, three-legged transformer.

The equations of the voltages on the primary and secondary are [5]

$$
\begin{aligned}
& v_{A}=R_{A}=i_{A}+L_{A A}=\frac{d}{d t} i_{A}+L_{A B}=\frac{d}{d t} i_{B}+L_{A C}=\frac{d}{d t} i_{C}+ \\
& L_{A a}=\frac{d}{d t} i_{c}+L_{A b} \cdot \frac{d}{d t} i_{b}+L_{A c} \cdot \frac{d}{d t} i_{c} \\
& v_{B}=R_{B}=i_{B}+L_{B E}=\frac{d}{d t} i_{B}+L_{B A}=\frac{d}{d t} i_{A}+L_{B C}=\frac{d}{d t} i_{C}+ \\
& L_{B a} \cdot \frac{d}{d t} i_{a}+L_{B \bar{b}} \cdot \frac{d}{d t} i_{b}+L_{B C}=\frac{d}{d t} i_{C} \\
& v_{\mathrm{G}}=R_{\mathrm{G}} \cdot i_{\mathrm{G}}+L_{\mathrm{GS}}=\frac{d}{d t} i_{\mathrm{F}}+L_{\mathrm{GA}}=\frac{d}{d t} i_{A}+L_{\mathrm{GB}}=\frac{d}{d t} i_{B}+ \\
& L_{C a}=\frac{d}{d t} i_{a}+L_{C b}=\frac{d}{d t} i_{b}+L_{C o} \cdot \frac{d}{d t} i_{c} \\
& v_{a}=R_{a}=i_{a}+L_{a A}=\frac{d}{d t} i_{a}+L_{a A}=\frac{d}{d t} i_{A}+L_{a B}=\frac{d}{d t} i_{B}+ \\
& L_{a c}=\frac{d}{d t} i_{c}+L_{a b}=\frac{d}{d t} i_{b}+L_{a c} \cdot \frac{d}{d t} i_{c} \\
& v_{b}=R_{b} \cdot i_{b}+L_{b b} \cdot \frac{d}{d t} i_{b}+L_{b A} \cdot \frac{d}{d i} i_{A}+L_{b B} \cdot \frac{d}{d s} i_{B}+ \\
& L_{b c}=\frac{d}{d t} i_{c}+L_{b a}=\frac{d}{d t} i_{a}+L_{b c} \cdot \frac{d}{d t} i_{c} \\
& v_{c}=R_{c}=i_{c}+L_{c c}=\frac{d}{d t} i_{c}+L_{c A}=\frac{d}{d t} i_{A}+L_{c o}=\frac{d}{d t} E_{B}+ \\
& L_{c c}=\frac{d}{d t} i_{c}+L_{c a}=\frac{d}{d t} i_{a}+Z_{c b}=\frac{d}{d t} i_{b}
\end{aligned}
$$

being $\mathrm{L}_{\mathrm{ij}}$ the self-inductance when $\mathrm{i}=\mathrm{j}$, and mutualinductance when $\mathrm{i} \neq \mathrm{j}$.

The above equations satisfy the following matrix relation:

$$
\begin{aligned}
& {\left[\begin{array}{l}
v_{A} \\
v_{B} \\
v_{c} \\
v_{a} \\
v_{b} \\
v_{c}
\end{array}\right]=\left[\begin{array}{cccccc}
R_{A} & 0 & 0 & 0 & 0 & 0 \\
0 & R_{B} & 0 & 0 & 0 & 0 \\
0 & 0 & R_{c} & 0 & 0 & 0 \\
0 & 0 & 0 & R_{a} & 0 & 0 \\
0 & 0 & 0 & 0 & R_{b} & 0 \\
0 & 0 & 0 & 0 & 0 & R_{c}
\end{array}\right]=\left[\begin{array}{c}
i_{A} \\
i_{B} \\
i_{c} \\
i_{a} \\
i_{b} \\
i_{c}
\end{array}\right]+} \\
& {\left[\begin{array}{llllll}
L_{A A} & L_{A B} & L_{A C} & L_{A a} & L_{A b} & L_{A c} \\
L_{B A} & L_{B D} & L_{B c} & L_{B a} & L_{B b} & L_{B c} \\
L_{c A} & L_{c B} & L_{c c} & L_{c a} & L_{c b} & L_{c} \\
L_{a A} & L_{a b} & L_{a c} & L_{a a} & L_{a b} & L_{a c} \\
L_{b A} & L_{b B} & L_{b c} & L_{b a} & L_{b b} & L_{b c} \\
L_{c A} & L_{c B} & L_{c c} & L_{c a} & L_{c b} & L_{c c}
\end{array}\right]=\frac{d}{d t}\left[\begin{array}{l}
i_{A} \\
i_{B} \\
i_{c} \\
i_{a} \\
i_{b} \\
i_{c}
\end{array}\right]}
\end{aligned}
$$

or in reduced form

$$
[\nu]=[R] \cdot[i]+[L] \cdot \frac{d}{d t}[i]
$$

To determine the current in each phase of the transformer the equation to resolve is

$$
\frac{d}{d t}[i]=[L]^{-1}([v]-[R] \cdot[i])
$$

For the determination of the magnetizing inrush currents are assumed previously deenergized transformer and no load on the secondary, so that when $i_{a}=i_{b}=i_{c}=0$ the system to solve becomes six to three equations

$$
\begin{aligned}
& v_{A}=R_{A} \cdot i_{A}+L_{A A} \cdot \frac{d}{d t} l_{A}+L_{A E} \cdot \frac{d}{d t} l_{B}+L_{A C} \cdot \frac{d}{d t} l_{C}(10) \\
& v_{B}=R_{B} \cdot i_{D}+L_{B D} \cdot \frac{d}{d E} i_{B}+L_{B A} \cdot \frac{d}{d t} i_{A}+L_{B C} \cdot \frac{d}{d t} i_{C}(11) \\
& v_{C}=R_{C} \cdot i_{C}+L_{C C} \cdot \frac{d}{d t} i_{C}+L_{C A} \cdot \frac{d}{d t} t_{A}+L_{C B}-\frac{d}{d t} i_{B}(12)
\end{aligned}
$$

simplifying the system matrices to solve.

\section{Self and mutual inductances determination}

The coefficients of the matrix [L] correspond to different values of the inductance and mutual inductance of the transformer coils. These parameters depend on the design of each transformer, which makes very complex a general determination.

However these parameters can be obtained for a real transformer by direct measurement of them. The selfinductance of each coil of the transformer can be determined from the relationship

$$
V=\sqrt{R^{2}+(\omega \cdot L)^{2}} \cdot I
$$

where $\mathrm{R}$ is the ohmic resistance of the winding, $\omega=$ $2 \cdot \pi \cdot \mathrm{f}$, the voltage $\mathrm{V}$ which is subjected to the winding and the current I circulating through the winding.

Similarly, the coefficient of mutual-inductance between two coils 1 and 2 can be determined from 


$$
V_{1}=w \cdot M_{12} \cdot I_{2}
$$

Both measurements are performed with the remaining windings in open circuit.

To test the proposed method, a real three-phase threelegged transformer 400/230 V and 1kVA was tested.

\section{Simulation results}

Figure 2 shows the simulation results during the first $100 \mathrm{~ms}$ considering constants and not saturable inductances, for a lag of the phase $\mathrm{R}$ voltage of 0.7 radians at the time of the connection.

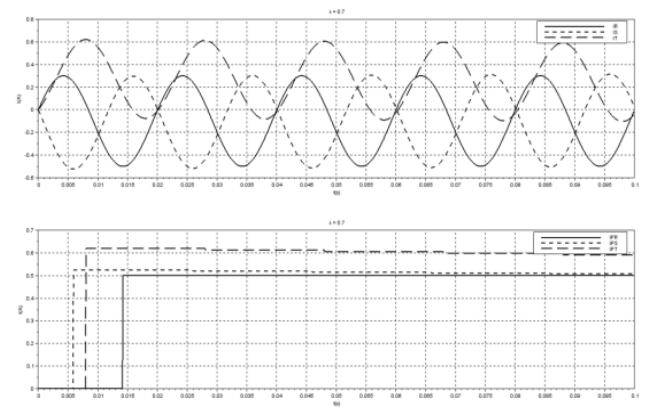

Fig. 2 Inrush currents and peak values without saturation

Figure 3 shows the peak values of the real inrush currents for the transformer studied.

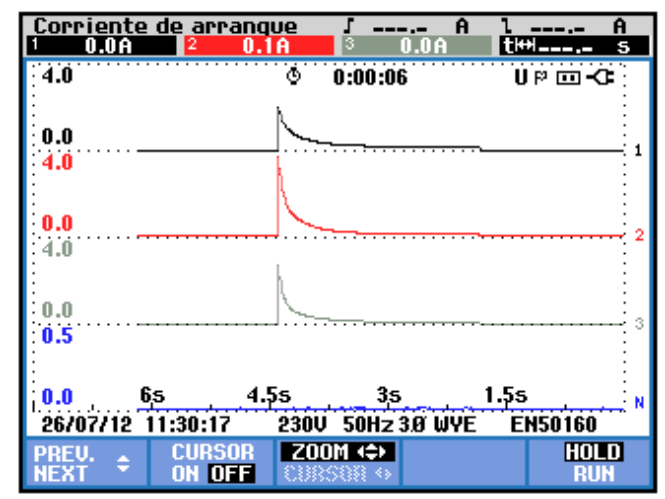

Fig. 3 Real inrush currents for 1 kVA transformer.

From the simulation, data shows that the simulation is quite inaccurate, as in the real case the peak current of the S phase is 3'6 A, and 0.62 A for the simulation. The reason for this is that the determination of the self and mutual inductance is not correct for the determination of the magnetizing inrush current, since in the initial instants these values are very high and it causes the saturation of the magnetic core [4].

To resolve this problem in a manner which is simple for the case of any three-phase transformer, we will be taken into account that the inductances are saturable, and therefore dependent on the current, $\mathrm{L}=$ $\mathrm{L}$ (i). For this, measurement is performed of the different inductances of the transformer taking pairs of current-voltage measurements from low currents to currents several times higher than the rated current of each coil.

The data so obtained form a set of measurement pairs of $\mathrm{I}-\mathrm{V}$ values is used to determine the induction as a function of the current using equations (13) and (14), taking $\mathrm{V}$ as the resultant value of the linear interpolation in the above set of pairs I-V values.

Since it is not possible to measure currents greater than 3 times the rated current to prevent damage to the windings during data collection, it is assumed that the core does not saturated more for values above that.

Once taken data and calculated the self and mutual inductances, the simulation is repeated, obtaining the data shown in Figure 4:
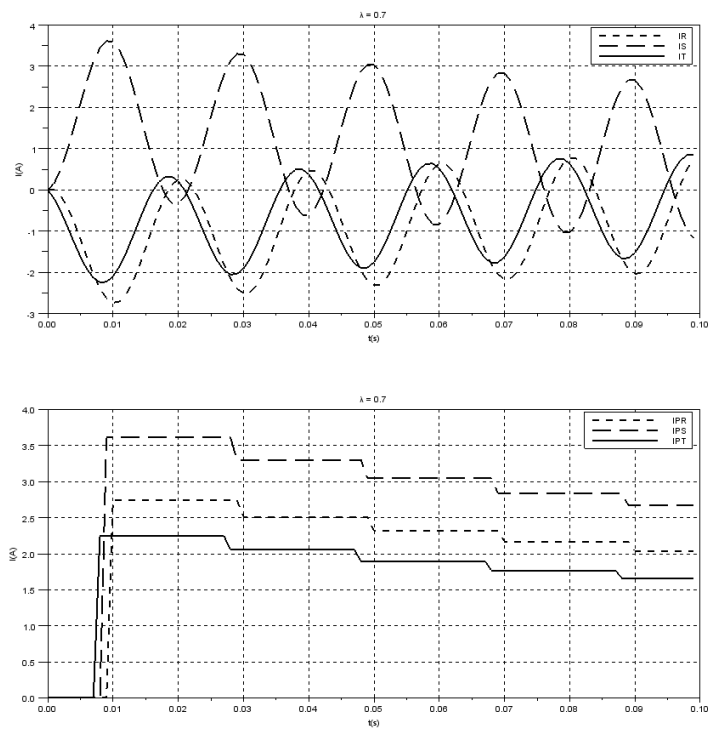

Fig. 4 Inrush currents and peak values with saturation.

Table 1 shows the comparison between the peak values for the three phases for the values obtained in the simulation and real data.

Table 1: Comparing real and simulated values

\begin{tabular}{|c|c|c|c|}
\hline Phase & Simulation & Real & Error \% \\
\hline R & 2'25 & 2'1 & 7 \\
\hline S & 3'61 & 3'60 & 0 '3 \\
\hline T & 2'74 & 2'66 & 3 \\
\hline
\end{tabular}

\section{Saturation and non linearities}

Calculation of self and mutual inductances is done for each coil separately, and applying superposition theorem. The data collection was done by applying increasing voltage to the coil and measuring both voltage and current for using with equations (13) and (14). 
Since voltage is applied from zero to maximum value, hysteresis is neglected. So there is no harmonics presence in the simulated currents.

Saturation of core is managed assuming that self and mutual inductances are current-dependant. So their value depends upon current flowing through the coil. For solving equation (9) the value of $\mathrm{L}_{\mathrm{ij}}$ is calculated for each iteration, for the differential equation solving; a linear interpolation of the data obtained by coils measurement is done and applying values to equations (13) and (14). Using this approach non linearities for self and mutual inductances are taken into account. Figure 5 shows the values of self and mutual inductances as a function of the flowing current. Inductance value is decreasing as flowing current rises. For low current values there is a lack of reliable data due to the sensibility of apparatus used for measuring, so in the simulation inductance are assumed to be constant for current values below a minimum.
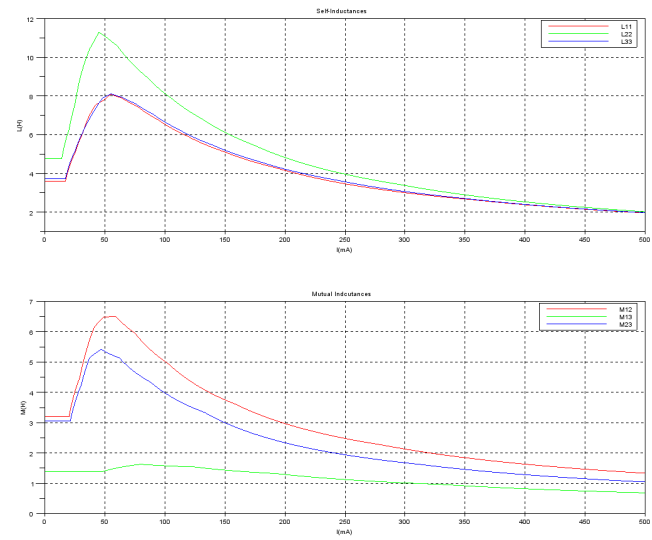

Fig. 5 Self and mutual inductances as a function of flowing current.

\section{Conclusion}

A method for determining the magnetizing inrush current for a three-phase, three legged transformer was proposed. The method uses as parameters, the coils resistance matrix and the coils inductance matrix. The resistances are measured directly in the windings of the transformer, and the inductances by obtaining pairs of current-voltage values from the inductances which are calculated as a function of the current through the coil. This data collection is extremely easy and achievable in any three-phase three-legged transformer.

The experimental results show that by using this method high precision is obtained in determining the peak value, the accuracy worsens by decreasing the value of the current, but it remains within acceptable limits.

The method takes into account the saturation of the core, but does not consider the phenomenon of hysteresis, which is why no harmonic currents are obtained by simulation. The latter makes this method not applicable to the simulation of inrush current for harmonics controlled differential protection of transformers, since the signals obtained from the simulation does not show the typical second harmonic in the magnetizing inrush current. However it is fully applicable to other protection systems that do not take into account the harmonic content of the signals, but their peak values and evolution.

\section{References}

[1] V. Marines Castillo, G. Idárraga Ospina \& E. Esteban Mombello. "Análisis de los Modelos de Transformadores para la Simulación de la Protección Diferencial". Ingenierías, Vol XIII, pp 65-75, march 2010.

[2] J. A. Martínez Velasco \& B. A. Mork. "Transformer Modelling for Simulation of Low Frequency Transients in Power Systems". $17^{\text {th }}$ International Conference on Electricity Distribution, session 1 document no 92, may 2003.

[3] M. A. Rahman \& A. GangoPadhyay. "Digital Simulation of Magnetizing Inrush Currents in Three-phase Transformers". IEEE Transactions on Power Delivery, Vol PWRD-1, n 4, pp 235-242, october 1986.

[4] Dongxia, Zanji \& Xiucheng. "Modelling and Simulation of Magnetizing Inrush Current of Large Power Transformers". $5^{\text {th }}$ International Conference on Electrical Machines and Systems, pp 440-443, 2001.

[5] Lin, Cheng, Huang \& Yeh. "Investigation of Magnetizing Inrush Currents in Transformers". IEEE Transactions on Power Delivery, Vol 8 no 1, january 1993.

[6] Abdulsalam, $\mathrm{Xu} \&$ Dinavahi. "Modelling and Simulation of Three-phase Transformers for Inrush Current Studies". IEE Proceedingns Generation, Transmission and Distribution, Vol 152, no 3, may 2005.

[7] M. P. Kostenko \& L. M. Piotrovsky. "Máquinas Eléctricas I". Ed. MIR. Moscow 1975.

[8] MIT E.E. Staff. "Circuitos Magnéticos y Transformadores”. Ed. Reverté 1973.

y

